\title{
MedChemComm
}

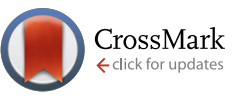

Cite this: Med. Chem. Commun., 2015, 6, 1370

Received 30th April 2015,

Accepted 12th June 2015

DOI: $10.1039 / \mathrm{c} 5 \mathrm{md} 00189 \mathrm{~g}$

www.rsc.org/medchemcomm

\section{Enhanced in vitro and in vivo uptake of a hydrophobic model drug coumarin- 6 in the presence of cucurbit[7]urilt:}

\author{
Xiaoqing Miao, ${ }^{a}$ Ye Li, ${ }^{a}$ lan Wyman, ${ }^{b}$ Simon M. Y. Lee, ${ }^{a}$ Donal H. Macartney, \\ Ying Zheng*a and Ruibing Wang*a
}

\begin{abstract}
This report describes, for the first time, cucurbit[7]uril-assisted quantitative in vitro and in vivo uptake of a hydrophobic model drug, coumarin-6, by both an epithelial cell model and a zebrafish model. The transcellular delivery pathway study suggested multiple mechanisms involved, including macropinocytosis, clathrin and lipid raft-mediated endocytosis/exocytosis.
\end{abstract}

High-throughput screening approaches in drug discovery and development have led to an increasing number of lipophilic drugs whose clinical usefulness is often hampered by their poor solubility in water, as water-solubility is one of the main factors to influence drug bioavailability. ${ }^{1,2}$ One of the popular approaches to improve the water solubility and bioavailability of drug candidates is to encapsulate them within macrocyclic molecular containers such as cyclodextrins, calixarenes, and cucurbiturils. $^{3-6}$ In particular, cyclodextrins have been studied for over 100 years and frequently used in pharmaceutical sciences to enhance the aqueous solubility of drugs and to improve drug bioavailability, e.g., several dozens of commercial pharmaceutical products based on cyclodextrins have been approved by regulatory agencies. ${ }^{3,7}$ Accordingly, the cellular uptake of both cyclodextrins and calixarenes, as well as the associated mechanisms, have been extensively studied. ${ }^{8,9}$ The cucurbit $[n]$ urils $(\mathrm{CB}[n], n=5-8,10,14)$, are a relatively new family of macrocyclic host molecules that have received increasing attention during the past 15 years. Consequently, investigations into their potential applications in the pharmaceutical sciences such as drug formulation and delivery are still far from real-world clinical use, presumably due to the scarcity of examples exhibiting both in vitro and in vivo uptake of a hydrophobic drug promoted by $\mathrm{CB}[n]$ and the lack of understanding of the associated transcellular mechanisms involved with $\mathrm{CB}[n]$-drug complexes. ${ }^{5,6,10}$

\footnotetext{
${ }^{a}$ State Key Laboratory of Quality Research in Chinese Medicine, Institute of Chinese Medical Sciences, University of Macau, Taipa, Macao, China.

E-mail: yzheng@umac.mo, rwang@umac.mo; Tel: +8538822 4689, +85388224687

${ }^{b}$ Department of Chemistry, Queen's University, Kingston, ON, K7L 3N6, Canada

$\dagger$ The financial support of this research by University of Macau and NSERCCanada is gratefully acknowledged.

\$ Electronic supplementary information (ESI) available. See DOI: 10.1039/ c5md00189g
}

Recently $\mathrm{CB}[n]$ hosts have demonstrated outstanding molecular recognition properties and superior interactions with a wide range of neutral and positively charged molecules, many of which are biologically- and medically-relevant compounds. ${ }^{5,6,11}$ Among the $\mathrm{CB}[n]$ family, $\mathrm{CB}[7]$ (shown in Fig. 1) has received perhaps the greatest attention as a potential drug delivery vehicle due to its well-studied biocompatibility profile, ${ }^{12-15}$ superior water-solubility and compatible size with various organic and organometallic drug molecules. $^{5,6,11}$ Examples of drugs that have been studied by us and by a few other research groups for their complexation behaviour with $\mathrm{CB}[7]$ include: a beta-blocker atenolol, ${ }^{16}$ a tuberculosis drug pyrazinamide, ${ }^{17}$ platinum-based anti-cancer drugs such as cisplatin and several others, ${ }^{18,19}$ local anesthetics, ${ }^{20}$ an anticoagulant drug coumarin, ${ }^{21}$ an anti-peptic ulcer drug ranitidine, ${ }^{22}$ and vitamin $\mathrm{B}_{12}$ as well as coenzyme $\mathrm{B}_{12} \cdot{ }^{23}$ Although these and other examples have exhibited enhanced water solubility as guest drugs upon $\mathrm{CB}$ [7] encapsulation, the actual benefit of such encapsulation during drug delivery (e.g. increased drug uptake), has not been clearly demonstrated with both in vitro and in vivo models. One previous study has reported the cellular uptake of $\mathrm{CB}[7]$ complexed acridine orange and pyronine by a mouse muscle embryo 3T3 cell model via fluorescence microscopy. ${ }^{24}$ Another study showed the uptake and trafficking of fluorescent dye tagged $\mathrm{CB}[7]$ with macrophage cell lines RAW264.7 by using flow cytometry and fluorescence microscopy. ${ }^{25} \mathrm{How}^{-}$ ever, the cellular uptake of these complexes was not

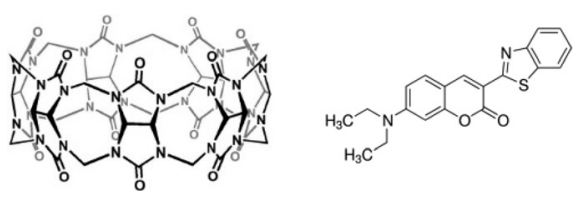

Fig. 1 The molecular structures of $\mathrm{CB}[7]$ (left) and $\mathrm{C} 6$ (right). 
compared with free guests (which can also cross cell membranes by themselves in both of these cases) in these studies. Thus the value of $\mathrm{CB}[7]$ as a delivery vehicle for the guest drug was not exhibited. One recent study demonstrated that $\mathrm{CB}[7]$ aided the transportation of a synthetic hemicyanine dye (Hsd) into living cells, thus facilitating selective RNA staining. ${ }^{26}$ However, the intracellular transportation mechanism of these complexes has never been studied previously.

Herein we report the $\mathrm{CB}[7]$-assisted cellular uptake of a hydrophobic model drug and a fluorescent dye, coumarin-6 (C6, Fig. 1), by a Madin-Darby canine kidney (MDCK) epithelial cell model. The MDCK cell line is a widely adopted epithelial model that is used to simulate biological barriers such as the gastrointestinal tract and blood brain barriers, and these barriers are often the main hindrance against the uptake of various substances in vivo, including new chemical entities and therapeutic agents. ${ }^{27}$ Thus it is critical to understand whether $\mathrm{CB}[7]$ can assist the endocytosis and exocytosis of encapsulated hydrophobic drugs with this epithelial cell model. In this communication, the benefits of $\mathrm{CB}$ [7] in the cellular uptake of a hydrophobic model guest drug by this important barrier tissue cell model was demonstrated for the first time. Importantly, it was found that $\mathrm{CB}$ [7] significantly improved the cellular uptake of the model drug. The transcellular transportation mechanism has also been studied in detail. Furthermore, the enhanced model drug uptake was further examined with a live in vivo zebrafish model.

C6 is a derivative of coumarin in the benzopyrone chemical class. It is a natural substance found in many plants, often used as a fluorescent and lipophilic model drug for the in vitro or in vivo monitoring of nanoparticle-based delivery systems via fluorescence or confocal microscopy. ${ }^{28}$ As a highly lipophilic model drug, it is not readily taken up by MDCK cells. ${ }^{27}$ As a matter of fact, the formation of a $1: 1$ guest-host complex between $\mathrm{C6}$ and $\mathrm{CB}[7]$ at neutral $\mathrm{pH}$ has been previously confirmed by ${ }^{1} \mathrm{H}$ NMR spectroscopy as well as UV-visible absorbance (Job's plot) and emission spectroscopy (titration), with a binding constant of $(2.0 \pm 0.1) \times 10^{3}$ $\mathrm{M}^{-1} .^{29}$ A MM2 energy-minimized structure of the C6@CB[7] complex (Fig. 2) shows that the benzopyrone portion is encapsulated within the $\mathrm{CB}[7]$ cavity, aligning the quaternary amine (protonated at neutral $\mathrm{pH}$ ) at one of its portals and leaving the benzothiazole ring outside of cavity. Such an encapsulation configuration is consistent with our previous
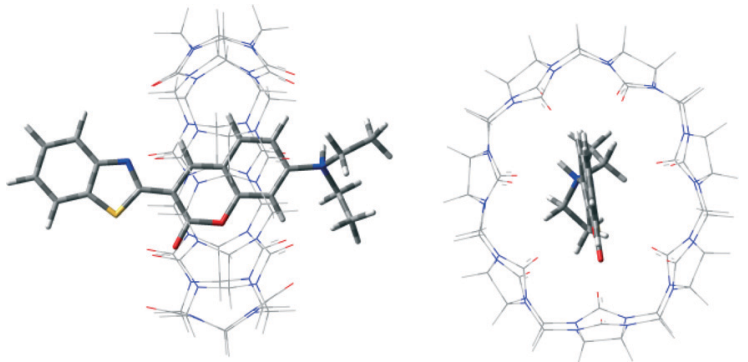

Fig. 2 Molecular modeling of C6@CB[7] (side view and top view). results from investigations focusing on the encapsulation of the parent coumarin by $\mathrm{CB}[7] .^{21}$

Prior to the in vitro and in vivo delivery study, the biocompatibility of $\mathrm{CB}[7]$ in the range of functional concentrations used in this work was examined with the MDCK cell lines (ESI $\dagger$ ). The results revealed that up to $1 \mathrm{mM}$ of $\mathrm{CB}$ [7] did not cause cell mortality. Thus $300 \mathrm{ng} \mathrm{mL}^{-1}(0.86 \mathrm{nM})$ of C6 in the absence and in the presence of $\mathrm{CB}[7](0.80 \mathrm{mM})$ were incubated with MDCK cell lines at $37{ }^{\circ} \mathrm{C}$ for 5, 15, 30, and 60 min. The large excess of $\mathrm{CB}[7]$ was added to ensure that the majority $(>60 \%)$ of the C6 species was in the bound form. The CB[7]-assisted intracellular delivery of C6 was monitored via fluorescence microscopy. As shown in Fig. 3, incubation MDCK cells with $\mathrm{C6} @ \mathrm{CB}$ [7] complex resulted in inclusion of green fluorescent $\mathrm{C} 6$ within the cells, and the drug was likely located in the membrane and endosome without obvious accumulation in the nucleus. Interestingly, the fluorescence intensity within the cells gradually decreased from $5 \mathrm{~min}$ to $60 \mathrm{~min}$, which is likely attributed to the initial $\mathrm{CB}[7]$ assisted C6 uptake in the complex form and subsequent release of free C6 in the cytoplasm (the fluorescence intensity of free C6 is much weaker in comparison with that of the $\mathrm{CB}$ [7]complexed form, ${ }^{29}$ as was seen from Fig. S1 $\dagger$ ). Detailed C6 distribution study by confocal laser scanning microscopy (ESI $\uparrow$ Fig. S3 and a video showing $z$ axis changes) confirmed that the $\mathrm{C} 6$ was mainly in the cytoplasm of the cells. In contrast, free C6 (either solubilized by Tween-80 or C6 suspension) exhibited little uptake by the MDCK cells regardless of incubation time. After the incubation treatment, the cells were rinsed three times with cold PBS and sonicated and ultra-centrifuged (13000 rpm for $30 \mathrm{~min}$ ) before the obtained supernatant was measured via fluorescence spectrophotometry. Interestingly the fluorescence emission resembled that of free C6, implying that C6 was released in the free form upon uptake by the MDCK cells. The C6 uptake therefore was quantitatively measured against its standard emission curve. The displacement of the included drug from $\mathrm{CB}$ [7] inside the cell environment is often desired for the purpose of

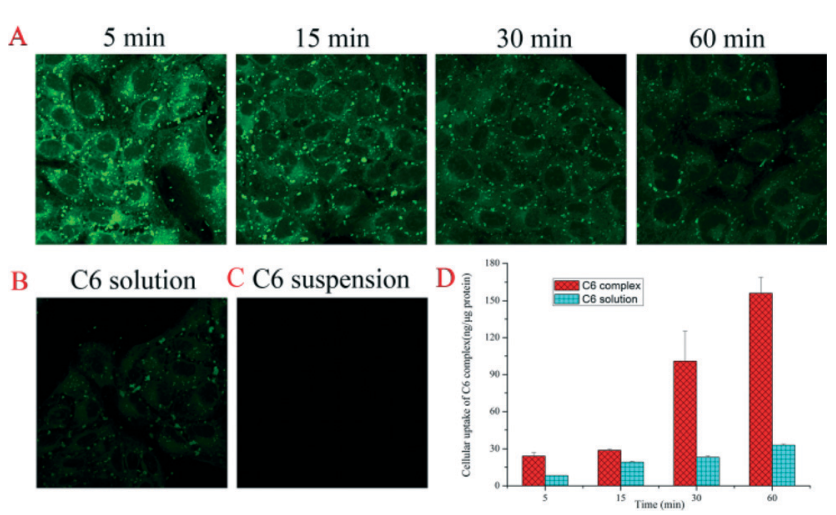

Fig. 3 Confocal laser scanning microscopy images of MDCK cell lines incubated for 5, 15, 30 and $60 \mathrm{~min}$ with $\mathrm{C} 6 \mathrm{aCB}$ [7] (A), $60 \mathrm{~min}$ with $\mathrm{C} 6$ solution (solubilized by Tween-80) (B), and 60 min with $C 6$ suspension ( 6 suspended in HBSS) (C). And the quantitative cellular uptake of $\mathrm{C} 6$ by MDCK in the presence of CB[7] and Tween-80 (D) $(n=3)$. 
intracellular drug delivery, and presumably this process occurs via competitive guest binding by protein residues (amino acids). ${ }^{30}$ Fig. 3D shows the performance of $\mathrm{CB}[7]-$ assisted cellular uptake of C6 quantitatively post incubation for different time-lengths. The cellular uptake increased gradually and reached a maximum at $\sim 1 \mathrm{~h}$. In the absence of $\mathrm{CB}[7]$, no detectable C6 was uptaken by the cells. Tween-80 was therefore added to enhance the solubility of $\mathrm{C6}$ and facilitate free C6 intracellular transportation process, ${ }^{31}$ for the sake of comparison. It seems that Tween-80 only moderately improved the cellular uptake of C6, which was still significantly less than the $\mathrm{CB}[7]$-assisted uptake. The significant benefit of $\mathrm{CB}[7]$ in the intracellular uptake of a hydrophobic model drug is clearly demonstrated quantitatively here for the first time.

In order to understand the cellular uptake mechanism and investigate how a $\mathrm{CB}[7]$-complexed hydrophobic drug would transport through an epithelial cell layer, endocytosis and exocytosis pathways were examined with several wellknown and commonly used transcellular regulators (Table S1 in $\mathrm{ESI} \dagger$ lists various inhibitors and the concentrations used in the study). For the endocytosis pathway detection, each of the regulators at a given concentration was pre-incubated with MDCK cells that were seeded in 12-well plates for 30 min. After the aspiration of the pre-incubated solutions, the C6@CB[7] complex (at the final concentrations of $0.86 \mathrm{nM}$ C6 and $0.80 \mathrm{mM} \mathrm{CB}[7])$ in HBSS and different inhibitors were added individually and further incubated at $37{ }^{\circ} \mathrm{C}$ for 30 min. This test was aborted by aspirating the dispersions and rinsing cells three times with cold PBS. The cells were subsequently scraped, washed and centrifuged according to the method described in the ESI, $\dagger$ before the supernatant sample was measured by fluorescence spectrophotometry. For the exocytosis pathway study, the MDCK cell monolayer was incubated in a similar manner with the same concentration of the $\mathrm{C6} @ \mathrm{CB}$ [7] complex at $37{ }^{\circ} \mathrm{C}$ for $30 \mathrm{~min}$ first, and then inhibitors that were dissolved in HBSS (listed in Table S1†) were individually added to re-incubate the samples with cells for another $30 \mathrm{~min}$ at $37{ }^{\circ} \mathrm{C}$ during exocytosis process. The intracellular fluorescent intensities of C6 were measured via fluorescence spectrophotometry. As shown by Fig. 4, filipin, nystatin, EIPA and M $\beta \mathrm{CD}$ exhibited pronounced effects on the cellular uptake of the complex during the endocytosis and exocytosis processes, thus implying that the endocytosis and exocytosis of the complex involved clathrin and lipid raft-mediation, as well as macropinocytosis during this process. Conversely, the brefeldinA and Monensin exhibited no effect during the transportation of the C6@CB[7] complex, thus suggesting that ER/Golgi and Golgi/PM pathways were not involved.

The study was further extended to a larval zebrafish model to examine $\mathrm{CB}[7]$-assisted in vivo uptake of the hydrophobic model drug. In recent years, zebrafish have emerged as useful vertebrate models for in vivo drug delivery including bioavailability and bio-distribution studies, due to the transparency of the whole body that enables non-invasive and direct

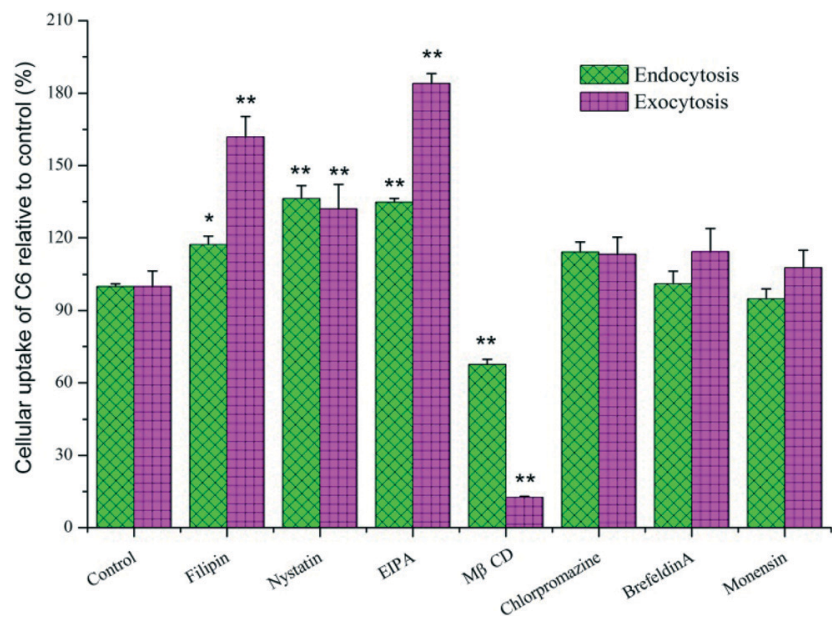

Fig. 4 Cellular uptake of $\mathrm{C} 6$ in the presence of $\mathrm{CB}$ [7] with various endocytosis/exocytosis regulators, as determined using ANOVA analysis in comparison with the control group $(* p<0.05, * * p<0.005$, $n=3)$.

observations, especially by optical tracking of fluorescent probes. ${ }^{32,33}$ Wild-type $\mathrm{AB}$ strain larval zebrafish at 8 days post fertilization were used for this study, as the structure and function of barrier and organs are similar to those of mammals. ${ }^{34}$ The zebrafish (30/group) were incubated with the C6@CB[7] complex with concentrations of 100, 200, 400 and $800 \mathrm{ng} \mathrm{mL} \mathrm{mL}^{-1}$ of 6 with a large excess of $\mathrm{CB}$ [7] (930 equivalents by molecular ratio) for $30 \mathrm{~min}$, respectively. Alternatively, the same concentration (300 ng mL ${ }^{-1}$ ) of $\mathrm{C6@CB[7]}$ was incubated for different time-lengths with the zebrafish samples. Equal quantities of the C6 suspension was added in the control groups, and Tween-80 solubilized C60 was added in groups of zebrafish for comparative purposes (ethical approval, detailed fish care, breeding, experimental and imaging protocols, are provided in the ESI $\dagger$ ).

As was the case with the in vitro results, no obvious fluorescence was observed in larval zebrafish treated with free C6 suspension, weak fluorescence was observed in the Tween-80 solubilized C6 (C6 solution) treated groups, whereas strong fluorescence was observed in the $\mathrm{C6} @ \mathrm{CB}[7]$ complex treated groups (Fig. 5). In addition, when all groups of fish were treated with the same concentration of C6@CB[7] complex, the fluorescence emission intensity increased significantly when the duration of the incubation was increased from 5 to $30 \mathrm{~min}$, and only increased moderately when the incubation time was increased from 30 to $60 \mathrm{~min}$, indicating an in vivo uptake likely reached its maximum between 30 and $60 \mathrm{~min}$ of incubation (Fig. 5B). Additionally, this time dependent uptake behavior was further confirmed by the uptake quantification results (Fig. 5D). Moreover, a dose dependent in vivo uptake of the $\mathrm{C6} @ \mathrm{CB}$ [7] complex was demonstrated as well (Fig. 5C). Higher concentrations of the C6@CB[7] complex resulted in enhanced fluorescence emission intensity within the treated fish body after $30 \mathrm{~min}$ of incubation, which is consistent with the quantitative uptake data shown in Fig. 5E. In contrast, free C6 was barely taken up by zebrafish 
A

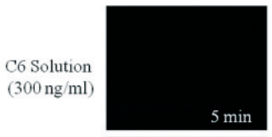

B
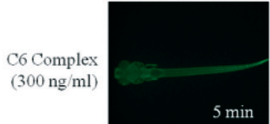

C
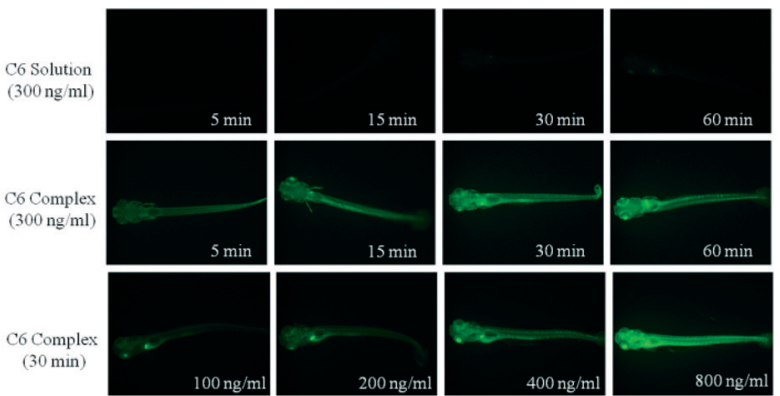

$\mathrm{D}$
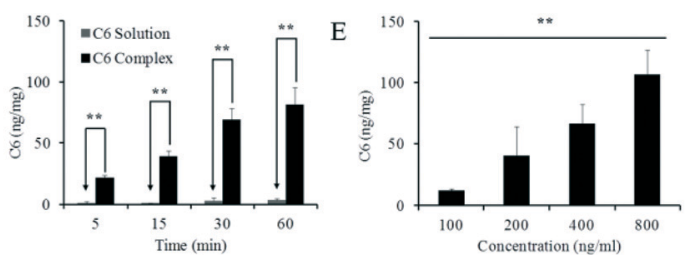

Fig. $5 \mathrm{CB}[7]$-assisted $\mathrm{C} 6$ uptake in a larval zebrafish model. In vivo fluorescence images of zebrafish larvae treated with $\mathrm{C} 6$ solubilized by Tween 80 (0.2 wt\%) at different time intervals (A), with C6 complex (300 $\mathrm{ng} \mathrm{ml}^{-1} \mathrm{C} 6$ in the presence of $0.80 \mathrm{mM} \mathrm{CB[7])} \mathrm{at} \mathrm{different} \mathrm{time}$ intervals (B), and with $\mathrm{C} 6$ complex at various concentrations (C). Quantitative uptake of $\mathrm{C} 6$ by larval zebrafish at different time intervals (D), and different concentrations (E). Each data point is the mean of three determinations, with error bar representing the S.D. $(n=3)$ $(* * p<0.01)$

and the addition of Tween-80 only slightly enhanced the uptake.

It is interesting to note that when the $\mathrm{C6@CB[7]} \mathrm{concen-}$ tration was at the lower range, the model drug preferentially targeted the eyes and the digestive system. Magnified fluorescent microscopic images of the zebrafish showed that the model drug preferentially accumulated in the following locations: the eyes, gall bladder, iridophores, and the microvascular system (Fig. S4 in ESI $\dagger$ ). After uptake reached maximum, the extracted supernatant (in embryo medium) from the zebrafish exhibited a single fluorescence band with a maximum at $500 \mathrm{~nm}$, which resembles the fluorescence spectrum of the free $\mathrm{C6}$ solution. This result suggested once again that the free model drug C6 was eventually released from the C6@CB[7] species after it was taken up in vivo (likely in a cellular environment), which is often desired for a drug delivery system, where a lipophilic drug is transported into the targeted area and released at this site to impose its pharmacological activity.

In summary, we demonstrate for the first time the $\mathrm{CB}[7]$ assisted quantitative in vitro and in vivo uptake of a hydrophobic model drug, by both an epithelial cell model (of a biological barrier) and a zebrafish model. The transcellular delivery pathway study suggested that multiple mechanisms were involved, including macropinocytosis, clathrin and lipid raftmediated endocytosis/exocytosis. This study provides critical evidence to support the use of $\mathrm{CB}[7]$ as a carrier for lipophilic drugs in order to enhance their bioabsorption and bioavailability. The C6@CB[7] system may also find applications in both in vitro and in vivo environments as a chemosensor, through competitive binding with other biologically important analytes. ${ }^{35,36}$

\section{Notes and references}

1 R. K. Verma, D. M. Krishna and S. Garg, J. Controlled Release, 2002, 79, 7-27.

2 S. Beg, S. Swain, M. Rizwan, M. Irfanuddin and D. S. Malini, Curr. Drug Delivery, 2011, 8, 691-702.

3 T. Loftsson and M. E. Brewster, J. Pharm. Pharmacol., 2010, 62, 1607-1621.

4 D. S. Guo and Y. Liu, Acc. Chem. Res., 2014, 47, 1925-1934.

5 S. Walker, R. Oun, F. J. McInnes and N. J. Wheate, Isr. J. Chem., 2011, 51, 616-624.

6 D. H. Macartney, Isr. J. Chem., 2011, 51, 600-615.

7 M. E. Brewster and T. Loftsson, Adv. Drug Delivery Rev., 2007, 59, 645-666.

8 A. I. Rosenbaum, G. Zhang, J. D. Warren and F. R. Maxfield, Proc. Natl. Acad. Sci. U. S. A., 2010, 107, 5477-5482.

9 R. Lalor, H. Baillie-Johnson, C. Redshaw, S. E. Matthews and A. Mueller, J. Am. Chem. Soc., 2008, 130, 2892-2893.

10 A. I. Day and J. G. Collins, Cucurbituril Receptors and Drug Delivery, in Supramolecular Chemistry: From Molecules to Nanomaterials, ed. P. Gale and J. Steed, John Wiley \& Sons, Ltd, 2012.

11 K. I. Assaf and W. M. Nau, Chem. Soc. Rev., 2015, 44, 394-418.

12 G. Hettiarachchi, D. Nguyen, J. Wu, D. Lucas, D. Ma, L. Isaacs and V. Briken, PLoS One, 2010, 5, e10514.

13 V. D. Uzunova, C. Cullinane, K. Brix, W. M. Nau and A. I. Day, Org. Biomol. Chem., 2010, 8, 2037-2042.

14 R. Oun, R. S. Floriano, L. Isaacs, E. G. Rowan and N. J. Wheate, Toxicol. Res., 2014, 3, 447-455.

15 H. Chen, J. Y. W. Chan, X. Yang, I. W. Wyman, D. Bardelang, D. H. Macartney, S. M. Y. Lee and R. Wang, RSC Adv. , 2015, 5, 30067-30074.

16 F. J. McInnes, N. G. Anthony, A. R. Kennedy and N. J. Wheate, Org. Biomol. Chem., 2010, 8, 765-773.

17 N. J. Wheate, V. Vora, N. G. Anthony and F. J. McInnes, J. Inclusion Phenom. Macrocyclic Chem., 2010, 68, 359-367.

18 N. J. Wheate, D. P. Buck, A. I. Day and J. G. Collins, Dalton Trans., 2006, 451-458.

19 N. J. Wheate, J. Inorg. Biochem., 2008, 102, 2060-2066.

20 I. W. Wyman and D. H. Macartney, Org. Biomol. Chem., 2010, 8, 247-252.

21 R. Wang, D. Bardelang, M. Waite, K. A. Udachin, D. M. Leek, K. Yu, C. I. Ratcliffe and J. A. Ripmeester, Org. Biomol. Chem., 2009, 7, 2435-2439.

22 R. Wang and D. H. Macartney, Org. Biomol. Chem., 2008, 6, 1955-1960.

23 R. Wang, B. C. MacGillivray and D. H. Macartney, Dalton Trans., 2009, 3584-3589.

24 P. Montes-Navajas, M. Gonzalez-Bejar, J. C. Scaiano and H. Garcia, Photochem. Photobiol. Sci., 2009, 8, 1743-1747.

25 G. Hettiarachchi, D. Nguyen, J. Wu, D. Lucas, D. Ma, L. Isaacs and V. Briken, PLoS One, 2010, 5, e10514. 
26 Z. Li, S. Sun, Z. Yang, S. Zhang, H. Zhang, M. Hu, J. Cao, J. Wang, F. Liu, F. Song, J. Fan and X. Peng, Biomaterials, 2013, 34, 6473-6481.

27 S. S. Zhao, W. B. Dai, B. He, J. C. Wang, Z. G. He, X. Zhang and Q. Zhang, J. Controlled Release, 2012, 158, 413-423.

28 C. Yu, B. He, M. H. Xiong, H. Zhang, L. Yuan, L. Ma, W. B. Dai, J. Wang, X. L. Wang, X. Q. Wang and Q. Zhang, Biomaterials, 2013, 34, 6284-6298.

29 N. Barooah, J. Mohanty, H. Pal and A. C. Bhasikuttan, J. Phys. Chem. B, 2012, 116, 3683-3689.

30 (a) M. A. Gamal-Eldin and D. H. Macartney, Org. Biomol. Chem., 2013, 11, 488-495; (b) J. W. Lee, H. H. L. Lee, Y. H. Ko, K. Kim and H. I. Kim, J. Phys. Chem. B, 2015, 119, 4628-4636.
31 R. G. Strickley, Pharm. Res., 2004, 21, 201-230.

32 K. Jobe, C. H. Brennan, M. Motevalli, S. M. Goldup and M. Watkinson, Chem. Commun., 2011, 47, 6036-6038.

33 Y. Liu, Y. P. Wu, D. F. Feng, Q. J. Ye, X. Yang, X. Liu, C. Y. Gao, M. Wu, D. Y. Chen, Y. J. Zhang, L. Li and X. Z. Feng, J. Mater. Chem., 2011, 21, 18704-18710.

34 C. Chakraborty, C. H. Hsu, Z. H. Wen, C. S. Lin and G. Agoramoorthy, Curr. Drug Metab., 2009, 10, 116-124.

35 G. Ghale, A. G. Lanctot, H. T. Kreissl, M. H. Jacob, H. Weingart, M. Winterhalter and W. M. Nau, Angew. Chem., Int. Ed., 2014, 53, 2762-2765.

36 C. Kim, S. S. Agasti, Z. Zhu, L. Isaacs and V. M. Rotello, Nat. Chem., 2010, 2, 962-966. 